\title{
Preparation and processing of human allogenic dermal matrix for utilization in reconstructive surgical procedures
}

\author{
Sarkozyova $\mathrm{N}^{1,2}$, Dragunova $\mathbf{J}^{2}$, Bukovcan $\mathrm{P}^{2}$, Ferancikova $\mathrm{N}^{1,2}$, Breza $\mathbf{J}^{3}$, Zilinska $\mathrm{Z}^{3}$, Koller $\mathbf{J}^{1,2}$ \\ Department of Burns and Reconstructive Surgery, Central Tissue Bank, Comenius University in Bratislava, \\ Faculty of Medicine, University Hospital Bratislava Ruzinov, Bratislava, Slovakia. koller.jan@gmail.com
}

\begin{abstract}
AIM: This article presents the development of a novel preparation and processing method as well as indication for clinical applications of human allogeneic acellular dermal matrix, which was developed originally in the Central Tissue Bank (CTB) for use in burn medicine and reconstructive surgery.

METHODS: Acellular dermal matrix (ADM) is a biological material assigned for utilization in several surgical procedures due to its unique structure and advantageous properties. The article focuses on a novel preparation and processing method developed by CTB, which differs in its impact on the structure, biological and biomechanical properties of the final ADM compared to the wide range of commercially available ADM products and currently available ADM products of other tissue banks.

RESULTS: The ubiquitous acellular allogeneic dermal collagen matrix is the main substance participating in advantageous properties facilitating the use of ADM in numerous indications from dermal replacement and soft tissue augmentation to more extensive surgical reconstructive procedures. Dermal substitutes play an essential role in the reconstruction of full-thickness skin defects, both in acute and chronic wounds, defects of fasciae, peritoneum, etc., and there is a strong evidence that they can improve the final scar quality as well. Differences in preparation methods of ADMs are recently causing concerns among surgeons utilizing the ADMs. We present three different cases with favourable outcomes by using human acellular ADM grafts. CONCLUSIONS: Although ADMs did not fulfil all of the requirements for an ideal dermal substitute, their applications have been advanced for diverse indications in soft tissue reconstructions and augmentations. Early revascularization of the allografts reduces bacterial contamination. Research and development of new generation of acellular dermal matrices with incorporated autologous in vitro cultured cells will likely yield new products and give new hope for continued improvements in functional and cosmetic outcomes (Fig. 9, Ref. 60). Text in PDF www.elis.sk.

KEY WORDS: human acellular allogeneic dermal matrix, skin and dermal substitutes, reconstructive surgery, wound healing.
\end{abstract}

\section{Introduction}

Major burns are extremely severe injuries with a high mortality. The advances in acute intensive burn care along with an early surgical treatment of deep burns including early excisions of necrotic tissues followed by coverage/closure of the excised areas in the 20th century resulted in a decreased mortality, and were often leading to functional and aesthetic late post-burn sequelae requiring secondary reconstructive procedures. Therefore, in the last decades, the main research did concentrate on the use of skin/dermal substitutes for scar hypertrophy prevention and management (1).

${ }^{1}$ Department of Burns and Reconstructive Surgery, Central Tissue Bank, Comenius University in Bratislava, Faculty of Medicine, University Hospital Bratislava Ruzinov, Bratislava, Slovakia, ${ }^{2}$ Department of Burns and Reconstructive Surgery, Hospital Bratislava Ruzinov, Central Tissue Bank (CTB), Bratislava, Slovakia, and ${ }^{3}$ Department of Urology, Renal Transplant Center, University Hospital - Derer's Hospital Bratislava, Slovakia

Address for correspondence: J. Koller, Prof, MD, PhD, Department of Burns and Reconstructive Surgery, Ruzinovska 4, SK-821 01 Bratislava, Slovakia.

Phone: +421.2.48234527
Dermal substitutes have been used to replace the architecture and function of the absent dermis in wounds with full-thickness skin loss. They are capable of revascularisation and promotion of re-epithelization in connection to the appropriate profile of bioactive molecules such as cytokines and growth factors and restoration both microarchitecture and function of the dermis in skin grafted areas. The large majority of the currently used dermal substitutes are decellularized collagen matrices of various sources either from human, porcine, fish or bovine origin (2). The next generations of dermal substitutes are tissue-engineered biomaterial dressings with complex bi-layered structure enriched by structural, signalling, cellular or tissue elements. The application of acellular dermal matrices has spread from the use in burn medicine to other surgical specialties dealing with chronic wounds, traumatic and contaminated wounds, and full thickness skin defects (3).

\section{Dermal substitutes}

Dermal substitutes are biological matrices that supersede functions of cutaneous dermal layer. Their presence in a wound bed reduces pain and radically decreases overproduction of scar tissue during the healing process. They act as matrices or scaffolds, they 
promote new tissue growth and facilitate wound healing with an enhanced pliability and a more favourable scars, thereby improving the long-term function and appearance of the healed skin related with a better quality of patients' lives $(1,4,5)$.

The loss of the dermis in extensive full-thickness skin wounds like major burns, skin ulcers or various deep wounds induces serious problems, which may be improved by application of a dermal substitute underneath the split-thickness autologous skin grafts thus mediating the wound healing process (6). Dermal substitutes are invaluable in reconstructive surgeries, while dermal tissue does not possess the ability of regeneration into the normal dermis in vivo after full-thickness dermal injuries $(1,7,8)$.

\section{Acellular dermal matrices}

Acellular allodermal matrices (ADMs) are prepared in tissue establishments from thicker split-thickness or full-thickness skin grafts of human origin procured from selected screened deceased donors. The process of procurement, preparation, processing and packing of ADMs is held under aseptic conditions. The skin grafts, either xenogeneic or allogeneic, may be freshly harvested or cryopreserved (10). The processing steps include enzymatic removal of the epidermis, thereafter the removal of all the remaining resident cells from the dermis (fibroblasts, macrophages, endothelial cells), resulting in a decellularized collagen-elastin based biomaterial matrix with antigenicity eliminated almost to none. The ADMs should retain the natural properties of the extracellular matrix (ECM) of which they consist of. They are immunologically inert, consisting of dermal collagen bundles together with preserved elastin fibres, proteoglycans, microvascular channels, and the complex of the basement membrane. ADMs are capable of permanent integration and revascularisation by the recipient organism (9). As ADMs are a material of biologic origin produced for implantation into the human body, there are several strict regulations for their preparation, processing, preservation, storage, distribution and clinical use.

\section{Material and methods}

\section{Basic principles of ADM preparation and processing}

Skin allografts following procurement are placed into sterile transport containers and transported to the accredited tissue establishment. Preparation and processing shall be provided in Class A clean rooms under aseptic conditions. The first step is the removal of the grafts from the containers and repeated rinsing in isotonic sterile solution in order to remove the debris from the grafts. The second step is decontamination of the grafts in a mixture of antibiotics solution. The process of decellularization is highly complex as the complete removal of any cellular and nuclear fragments out of the ECM requires multiple steps. In the past, there have been several techniques used for the processing of the grafts to ADMs based on different methods. Depending on the skin graft sources, different approaches of decellularization have been developed. The initial techniques utilized a combination of a proteolytic enzyme (trypsin), freeze-thawing and long-running incubations causing autolysis of cells by enzymes presented in the graft. Their drawbacks included a high price and potentially toxic residues left in the dermal matrices, that could cause structural damage to the remaining dermal matrix and inhibition of cell proliferation during their incorporation. After implantation, these pioneer ADMs might induce an immune response of the host at some level that could result in a poor graft survival due to its rejection (11-13). Besides, any impairment either to the collagen or the non-collagen proteins, growth-factors and glycosaminoglycans may lead to a strong inflammatory reaction with a subsequent lysis and resorption of the implanted ADM (11). Nevertheless, the preservation of an intact ECM architecture is crucial for the characteristics of the final ADMs.

\section{Currently used processing methods for ADM preparation Physical methods}

The dermis is a quite thick and dense material, therefore it needs a preliminary treatment prior to the application of chemical reagents that penetrate through the pores to start the diffusion of the reagents. Generally applied methods as pressure, ultrasound or thermal shock have been used to accelerate the decellularization of the tissues and organs. Commercially prepared ADMs have been processed by protocols using sonication and agitation in conjunction with enzymatic and chemical methods. (14)

\section{Chemical methods}

The chemical methods include treatment with alkaline or acid media, detergents, immersion to hypotonic or hypertonic solutions, and chelating agents. These methods had to be used in combination with other methods to increase the decellularization efficacy (15). Biologic methods

Proteolytic enzymes have been used for decellularization of various tissues, however, in decellularization of the dermis the collagenases should be avoided as they may break the peptide bonds in the collagen bundles thus damaging or destroying the matrix structure. These methods should be used in conjunction with the other treatment methods mentioned above in order to optimize cell removal (15).

\section{Decellularization criteria}

To classify tissue as acellular, the methods quantifying the presence of nuclear material from cell residues present within the ECM are used. The amount of dsDNA (double stranded DNA) per $1 \mathrm{mg}$ of ECM in dry mass should not exceed $50 \mathrm{ng}$, the acceptable length of DNA fragments is less than $200 \mathrm{bp}$ (base pairs) (16). Light microscopy examination is advised to prove the absence of any nuclear material in tissue sections stained with haematoxylin and $\operatorname{eosin}(11,17)$.

\section{The novel method for ADM preparation developed in the CTB}

Since the only currently available ADMs have been prepared and distributed only commercially, where the costs were far over the available burn department budget, the researchers from the burn department and the Central Tissue Bank of the Burn Department in Bratislava have developed a novel, simple, and economic method for the preparation of ADM. The new method combines the initial enzymatic treatment using trypsin together with the exposure to a hypotonic medium. Skin grafts assigned for ADM preparation and 
processing have been prepared, processed and packed in cleanrooms of the Central Tissue Bank (CTB) of the Ruzinov University Hospital according to approved standard operation procedures by the tissue bank personnel (18).

\section{Decontamination}

Decontamination of the grafts is provided by a repeated rinsing in a mixture of antibiotics solution. (18)

\section{Decellularization}

The first step is the exposure of the allogeneic or xenogeneic split-thickness skin grafts to the co-mixture of trypsin and ethylenediamine tetraacetic acid (EDTA), which results in the separation of the epidermal layer from the intact basement membrane. Consequently, the epidermal remnants are removed by mechanical scraping, and subsequently, the epidermis-free dermal grafts are submerged into a hypotonic solution medium (sterile distilled water bath), where step by step lysis of the resident dermal cells occurs due to a low osmotic pressure environment. Rinsing of the grafts in the hypotonic solution is repeated several times leaving an intact acellular dermal collagen-elastin matrix with a retained structure. The overall time of preparation including the last phase of decontamination takes 7 days (18).

Acquiring samples for microscopic and bacteriology investigation

From each batch of the processed ADMs, random small samples $(1 \times 1 \mathrm{~cm})$ are taken for microscopic and microbiologic investigations. The samples for bacteriology are sent to microbiology laboratory accredited for sterility testing according to European Pharmacopeia (18).

Packing and storage

The final products - ADM sheets are placed in pre-labelled cryoprotective sterile plastic bags and stored in quarantine deep freezers at $-70^{\circ} \mathrm{C}$. After the negative results of the obligatory serology tests of the donors and the results of the sterility tests are obtained, the ADM grafts can be released for clinical use. The overall storage time shall not exceed 5 years (18).

\section{Results}

Histology, residual DNA analysis, testing for cytotoxicity and biocompatibility (in vitro cultivated cells growth curve)

Histology

During the decellularization procedure, allodermis samples from each step have been taken and fixed by buffered $10 \%$ formalin solution. The samples were cut by microtome, spread and fixed on glass slides, stained by haematoxylin-eosin and prepared for microscopy at the Department of Pathology (Mrázová et al, 2014). Analysis of DNA content DNA was isolated using GeneJET Genomic DNA Purification Kit (Thermo Scientific) according to the protocol provided by the manufacturer (19).

Residual DNA analysis

The DNA analysis test was performed at the Institute of Medical Biology, Genetics and Clinical Genetics, Faculty of Medicine, Comenius University in Bratislava. The number of samples tested for DNA content was 5 from cryopreserved grafts and 5 from fresh grafts. The negative results from both groups were identical (19).

\section{Cytotoxicity testing}

Three different cytotoxicity tests were performed for cytotoxicity evaluation.

Agar diffusion test

It was performed according to the standard method used in the (CTB) (Vittekova et al, 2014). Briefly, the tested samples of ADM $(0.5 \times 0.5 \mathrm{~cm})$ were placed into a Petri dish covered with in vitro monolayer culture of cells - human dermal fibroblasts (HDF), or mouse 3T3 cells. After $24 \mathrm{~h}$, the content of the Petri dishes was stained by neutral red agar medium (Difco Bacto agar). The potential toxic zone was observed during 3 consecutive days. AlloDerm (LifeCell Corporation, The Woodlands, Texas, USA) and sterile gauze was used as negative controls. Different acellular dermal matrix- Xeno-UK (Institut Biomedicinych technologij Ternopil, State Medical University, Ukraine), was used as a control with a semi-toxic effect (Vittekova et al, 2014). Gauze soaked in $20 \%$ SDS (Sodium dodecyl sulfate) was used as a positive control (19). Contact cytotoxicity test

Tested samples were cut into $0.5 \times 0.5 \mathrm{~cm}$ pieces and placed into sterile Petri dishes for tissue cultures. The samples were fixed on the bottom of the dishes and, subsequently, a suspension of live cells (HDF or mouse 3T3 cells) was added. An average number of cells seeded into dishes was $0.3 \times 10^{6}$ /Petri dish. Morphological changes were observed microscopically on each day of cultivation. The positive, negative, and semi-toxic controls were identical to the ones used in the agar diffusion testing (19).

In vitro cultivated cells growth curve

The growth curve of the cells cultured in the presence of tested scaffolds was estimated. The scaffolds $(0.5 \times 0.5 \mathrm{~cm})$ were placed in Petri dishes, covered with the HDF or $3 \mathrm{~T} 3$ cell suspensions $\left(0,3 \times 10^{6} /\right.$ Petri dish $)$. The dishes were cultured in a $\mathrm{CO}_{2}$ incubator at $37^{\circ} \mathrm{C}$. After $24 \mathrm{~h}$ of incubation, the cells were trypsinized (Vitteková et al, 2014) and the cell numbers were counted microscopically in Burker's chambers. For each parallel, the cell numbers of 3 Petri dishes for every scaffold were calculated. The cell counting was performed at $24,48,72,94$, and $168 \mathrm{~h}$. The positive, negative and semi-toxic controls were identical to the controls used in the contact cytotoxicity testing (19).

\section{Biologic reactions in the host body}

Preparation methods of ADMs determine the host reaction as well as the final outcome of surgical procedures. The biologic response may be classified as acceptance with integration and nonacceptance of the ADM (11).

The mechanism of ADM integration is the subject of further investigation. There are three theories of possible integration that support the remodelling of the ultrastructure of the implanted ADMs. The first mechanism follows after a biodegradation of large insoluble molecules of ECM of ADM in vivo, where lesser fragments - cryptic bioactive peptides with different biologic characteristics are released into the environment. Another way is the chemotaxis of endogenous progenitor and stem cells into the ECM of implanted ADM. The last mechanism is the modulation of immune response, when the host organism produces the M2Th2 phenotype of lymphocytes (20). 
Only 12 hours directly after implantation, the ADM is populated by circulating cells, which establish a neovasculature and elaborate new extracellular matrix, while incorporating the ADM material. In 7 days, small vessels are present, mass density decreases up to 60 days post-implantation, when mature vessels with a larger calibre and thicker walls are present (21).

ADMs processed by unsparing methods including sterilization, crosslinking and conservation suffers damage to the ultrastructure of the scaffold, which leads to an intensive fibrotic response of recipients organism. After incorporation and influx of host cells, the implanted ADM is detected as foreign material and the ADM, depending on the intensity of inflammatory reaction, is degraded with the formation of scar tissue and deposition of dense tissue.

Non-acceptance and non-incorporation of ADM is a reaction of the host organism, terminating into absence of any influx of host cells into the scaffold, which is recognized as a foreign material. This reaction leads to the persistent inflammatory impetus conducts to a chronical inflammatory response with a production of dense tissue encapsulating the ADM (11).

\section{The final ADM product}

The final ADM product was a dermal matrix with preserved essential biochemical and structural properties of the human dermis. It was proved to be non-antigenic, in contradiction to the skin allograft, which is antigenic due to the presence of cellular structures $(3,22)$. The preparation method used proved to be highly efficient, relatively fast and did not leave any toxic residues in the final ADM with the preserved natural structure of the ECM. Such ADM proved to be suitable not only for clinical applications, but on its account of low cytotoxicity also for cell cultivations as a carrier for in vitro cultured cells for regenerative medicine (18).

\section{Clinical applications and indications of ADMs}

The applications of ADMs in reconstructive surgery have become quite common over the past 20 years. ADMs are currently far more used in reconstructive surgery than in burns, as a soft tissue implant for augmentation and reconstruction of different body areas such as: tympanic membrane (23), cleft palate (24), dura mater (25), nose (26), lips (27), chest (28), abdominal wall (29), upper and lower extremities $(30,31)$, pelvis etc. $(32,33,34)$. Many of these reconstructive surgical interventions have been performed using autologous, allogeneic, xenogeneic, or synthetic/ biosynthetic allogeneic or xenoplastic materials. Autologous tissues, such as fat, dermis, fascia, cartilage, bone, and muscle, hold the precedence over heterologous or allogeneic materials if they are available to be harvested in sufficient amounts without undue trauma to the donor. However, often the available autograft is insufficient or causing significant trauma to donor-site, which is unacceptable. Alloplastic materials may suffer from poor tissue adherence, may elicit a foreign-body reaction, and may be at risk of extrusion (35). Xenogeneic collagen is often used as well, however, the material is rapidly resorbed and may cause an antigenic reaction (36). These are the main reasons, why the allogeneic materials are in a superior position.
Processing methods of ADM may determine the host reaction and thus determine the final outcome of the surgical procedures. The biologic response may be classified as acceptance with integration and/or non-acceptance of the ADM implant (11). The exact mechanism of ADM integration is a subject of further investigation.

Once the ADM is repopulated by host cells and revascularized from surrounding host tissue, the integration can lead to lower infection rates than for synthetic materials (31). In addition, the bioscaffold has been shown to promote cell migration, proliferation, and vascularization, and, therefore, it accelerates wound healing $(37,38)$. ADM graft is ultimately remodelled into a tissue with the characteristics of the site into which it has been implanted. However, while implanted to treat deep skin defects, having no epidermal layer, the substitute itself provides a limited barrier function only. When used as a graft, it must be covered with a thin split-thickness skin autograft as well as a moistureretaining dressings (39). Autologous full-thickness skin grafts may fail due to high metabolic demands, therefore ADM has an advantage as their metabolic demands raise gradually with cell infiltration and revascularization, and just after the full integration they are finished. The nutritional requirements are the same as in the surrounding tissues (40).

Composite skin grafting

In contrary to epidermis, after suffering superficial dermal injury, any defect heals by reparation instead of regeneration. If collagen components are missing, the whole process leads to deposition of scar tissue. Examples of such healing are deep burns and extensive full-thickness skin defects. The golden standard in the treatment of such wounds is an application of split-thickness skin graft (STSG), which, however, leads to the formation of contraction scars. The substitution of missing dermal components may be accomplished by the application of a dermal substitute, such as $\mathrm{ADM}$, that reduces the formation of scars, as well as the wounding of the donor sites (11).

In addition, ADM creates a better wound-bed microenvironment, increasing the healing rate of the combination of ADM and STSG. Another important advantage of composite skin grafting is, that ADM as the scaffold continues to facilitate wound healing and skin graft acceptance (41-43). The elastic fibre structure of the ADM has an excellent resilience for the prevention of scar formation. At the same time, the ADM has significant haemostatic properties and might reduce the risk of hematoma formation under the grafted skin $(44,45)$. Before the application of ADM into the wound bed it is suggested to perforate or mesh the ADM to prevent seroma or hematoma as well as to enhance the adhesion of ADM to the uneven wound bed surface.

If negative pressure wound therapy (NPWT) is applied, the composite skin grafting may be performed as a one-stage operation. The NPWT applied over the composite skin graft reduces the risk of hematoma or seroma formation, moreover, it supplies its fixation in the wound bed, improves microcirculation and oxygenation of surrounding tissues and therefore reduces the need for another operation, which always represents possible complications $(46,47)$.

In our experience, the application of composite skin grafts was highly useful, when tendons and deep structures were exposed. 


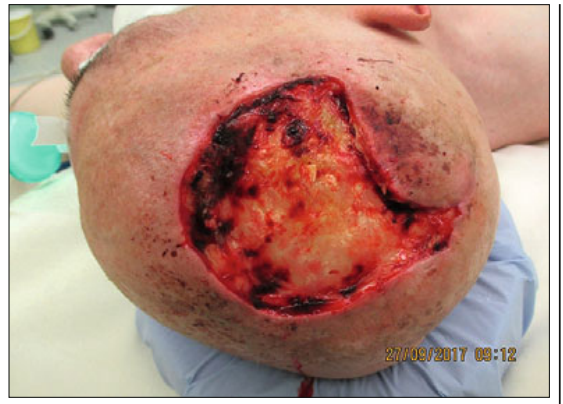

Fig. 1. Cranial defect after debridement, with exposed dura mater.

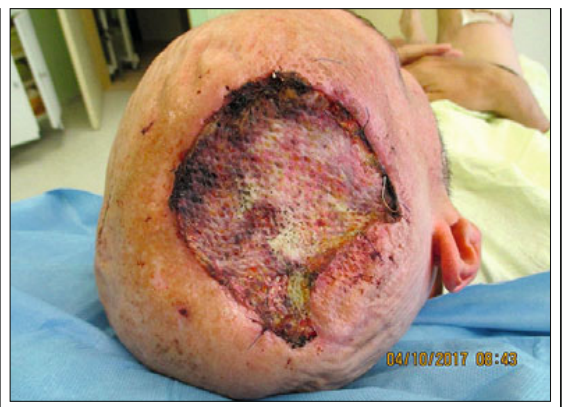

Fig. 2. 8th post-op day, present revascularization and intake of composite graft.

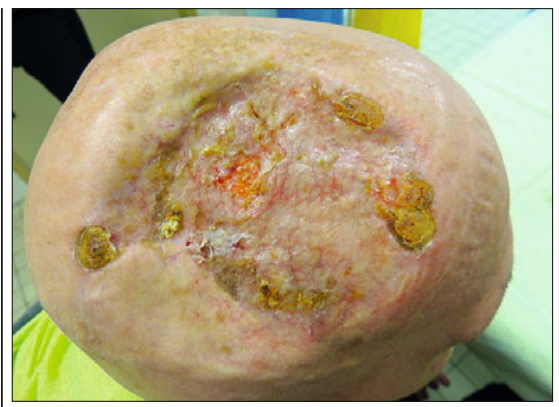

Fig. 3.1 year post-op, matured, well-vascularized composite graft.
Transplanted composite skin showed better outcomes than STSG alone, showing no or almost no signs of contracture. Such skin was pliable, softer, and with better final aesthetic outcomes.

ADM in soft tissue reconstruction and augmentation

The application rate of ADM in reconstructive surgery procedures is expanding. The indications for ADM applications include (a non-exhaustive list) dermal replacement in extensive burn injuries, hypertrophic and contracted scars revisions, breast surgery, peritoneal and abdominal wall defects reconstructions, etc.

The use of ADM in implant-based breast reconstructions became over the last decade the gold standard. This method has been popularized as an adjunct to implant-based surgery given its utility in providing coverage of the implant and support for the inferior pole of the breast as well as it prevents capsular contracture formation (48). Another applications with positive outcomes proved to be reconstructions of the disrupted abdominal walls following a repeated laparotomy wounds dehiscence complicated by infection $(49,50)$.

\section{Risks of ADM applications}

Allogeneic ADM is a tissue of human origin, accordingly, there is always a possible threat of disease transmission. To our knowledge, there have been so far no known cases of infection transmitted by ADMs. The protocols in tissue banks for the preparation of ADM adhere to a strict donor screening and acceptance criteria. The tissue establishments are obliged to follow regulatory guidelines and keep records. The risk of disease transmission by xenogeneic material is believed to be very low (3).

\section{Clinical study - outcomes}

Hereby we would like to briefly present 3 cases, where this unique biomaterial was used in various indications in patients treated at our department. In all the cases, aseptic ADM was implanted in one-stage operation with the application of $1: 1$ meshed allogeneic ADM into prepared wound bed overlayed by a split-thickness skin autograft or with closing the wound by a primary suture.

\section{Case 1: Cranial defect without bone and skin cover with a leakage of cerebrospinal fluid coverage}

43-year-old cancer patient in remission with a cranial defect in the temporo-parietal region after the explantation of an infected methacrylate bone implant (KIT) complicated by a skin necrosis in the implant site. On September 20th, 2017 the KIT implant was extracted and following a thorough wound debridement, a temporary coverage of the defect with allodermis was provided. 7 days later, a permanent coverage of the defect with an exposed dura mater with a composite graft of 1:1 meshed ADM covered by autologous split-thickness skin graft was performed. At 1 month post-operation, more than $90 \%$ of the composite graft was revascularized, only small defects covered with dry crusts were present at the edges. At 1-year post-operation, the whole transplant was well vascularized, the skin graft has got matured. Minimal residual fistulae with cerebrospinal fluid leakage were still present (Figs 1, 2, 3).

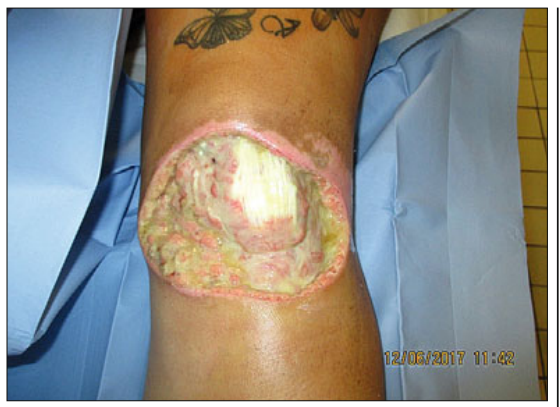

Fig. 4. Deep traumatic defect in the pre-patellar region, before debridement.

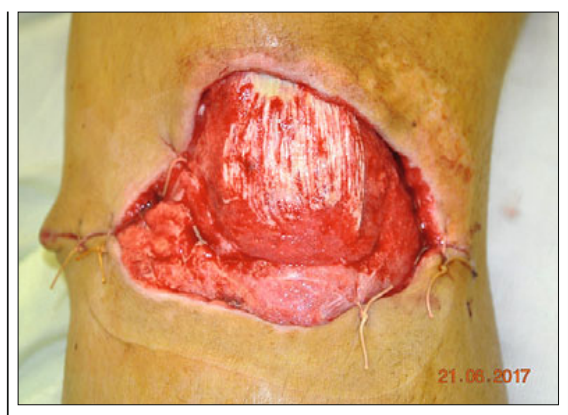

Fig. 5. The defect after 2 debridements, ready for permanent closure.

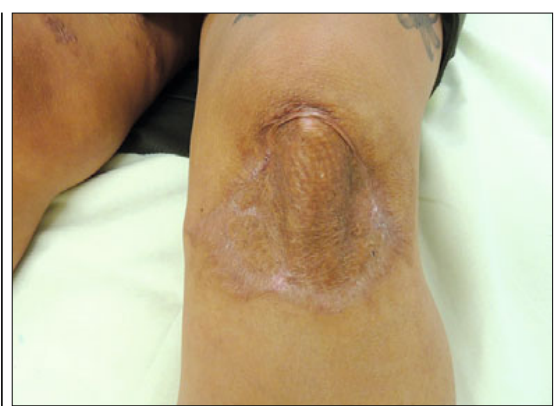

Fig. 6. Engrafted composite graft, without contracting scar formation. 


\section{Case 2: Deep post-traumatic knee defect reconstruction}

28-year-old patient after a motor-bike accident with a devastating-laceration injury in the left prepatellar knee area with exposed ligaments and full-thickness skin defect. During the revision of the defect, the non-viable parts of the skin cranially and on both sides were found. Debridement of the non-viable and potentiallyviable soft tissues together with a superficial partial excision of the patellar ligament was performed. For faster and better wound bed preparation a negative pressure wound therapy (NPWT) device was applied. A second debridement after 5 days was performed. Permanent closure with a bilaminar technique (ADM overlaid with a thin skin autograft) was performed on the 8th day after the first operation. The composite graft got well-vascularized. The transplanted skin, even in this difficult anatomical region 3 years post-operation, is still durable and flexible without the formation of contracting scars. Full extension and flexion of the knee joint are present (Figs 4, 5, 6).

\section{Case 3: Abdominal wall reconstruction with ADM for ex- posed kidney graft salvage}

54 years old poly-morbid renal transplant patient with a repeated post-operative wound dehiscence and exposure of the transplanted kidney. The surgical management required two surgical interventions. The first one was a thorough surgical debridement of the open abdominal wound along with the undermined wound edges followed by a temporary protective coverage of the exposed kidney by human skin allograft and application of NPWT. The debrided and prepared wound was closed after a skin allograft removal by ADM used for both the transplanted kidney protection and reinforcement of peritoneal and abdominal fascia defects as a biologic net. Following peri-renal and subcutaneous space drainage, the abdominal wound was closed and healed within 22 days. At 6 months follow up, a complete wound closure with a firm scar and no abdominal herniation along with a good transplanted kidney function have been found (Figs 7, 8, 9).

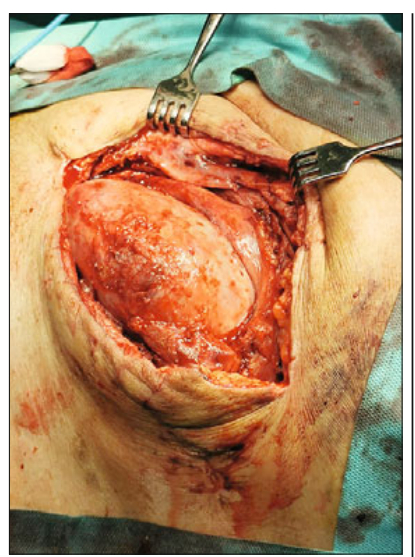

Fig. 7. The disrupted abdominal wound with an exposed kidney, before the first operation.

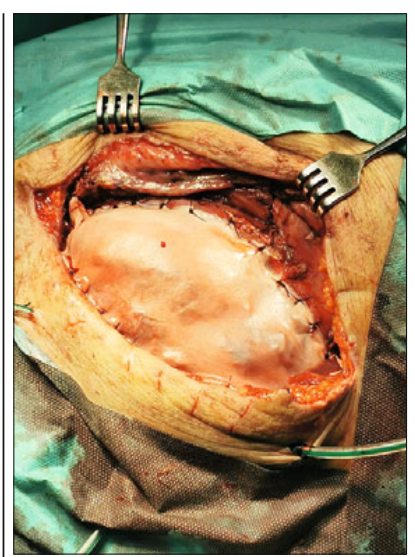

Fig. 8. The debrided wound, with a prepared wound bed, the exposed kidney was covered by ADM, the edges sutured to healthy fascia.

\section{Discussion}

The differences in ADMs: xenogeneic vs allogeneic, sterile vs aseptic preparation

Acellular dermal matrices are prepared either from xenogeneic or allogeneic material. The obtained xenogeneic tissues assigned for decellularization came as side line products. Xenogeneic tissues are either bovine, porcine, equine or fish origin (40). There is a strong evidence for differences in biologic reactions to implanted different types of ADMs. Xenogeneic matrices, even after decellularization, retained an alfa-gal epitope, which did not occur in human body proteins. Therefore, the application of xenogeneic ADMs did lead to negative interactions with antibodies, resulting in a localized inflammation and resorption of the matrix (51).

Other differences causing concerns among surgeons utilizing ADMs are in preparation methods. The aseptic ADMs have been processed under aseptic conditions in a sterile environment avoiding secondary contamination. To avoid terminal sterilization, which causes ultrastructural changes in molecules of the ECMs, aseptic processing relies upon antibiotics alongside the decellularization process. Aseptic processing with an intact natural ultrastructure of ECM confers a sterility assurance level of $10^{-3}$. The sterile ADMs undergo sterilization by gamma irradiation reaching a sterility level of $10^{-6}$, however, causing structural changes in the ECM (52). The processing methods determine the occurrence of the final post-implantation biological reaction in the recipient organisms.

\section{Requirements for the dermal substitutes}

Intact ultrastructure of dermal ECM

Implanted ADMs due to their scaffolding properties restore the microarchitecture and physiologic functions of the reconstructed areas (7). The acellular matrix preserved intact dermal vascular channels that could be repopulated by the recipient's endothelial cells leading to a rapid revascularization. The vascularization period of the dermal component took place usually around 21 days. Vascularization of the ADM is crucial to enhance the take of split-skin grafts and to assure a primary healing

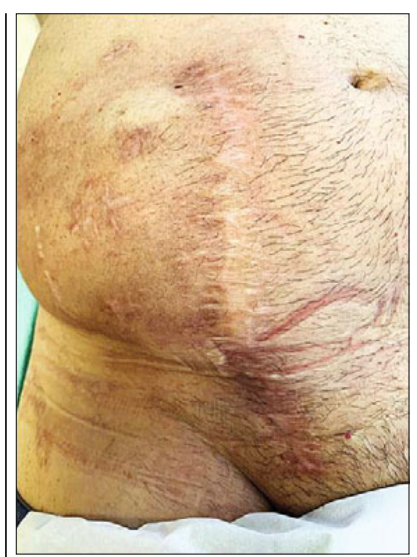

Fig. 9. Healed reconstructed abdominal wound, 6 months postoperation. with a solid scar in soft tissue reconstructions (53-55). Even though the new ADM scaffold material was described as "permanent", generally, it is considered that the transplanted scaffold will be repopulated by the host dermal cells capable to resynthesize a new autologous dermal matrix $(1,8)$.

Biocompatibility

The main characteristics distinguishing ADMs from other materials include a tissue integration, host tolerance, and immunologic compatibility. ADMs are biomaterials consisting mostly of collagen scaffolds, 


\section{6-394}

which are just in rare cases immunogenic, therefore they are an ideal material for implantation as only a few individuals have an inborn immunogenic reaction against allogeneic collagen molecules. Insufficient removal of any immunogenic materials, such as cells, cell debris, chromosome fragments, etc., from cells that express foreign major histocompatibility complex antigens, would initiate both innate and adaptive immune responses leading to a rejection as in unprocessed skin grafts used for temporary dressing (11). Biocompatibility was demonstrated by the early ingrowth of fibroblasts and blood vessels (56). Apart from a rapid adherence and vascularization, other related factors taken into consideration were mechanical stability and durability (57).

Non-toxicity and biodegradation

The main advantage of collagen-based materials as ADMs, is their biodegradability and biocompatibility. Both are related to a unique structure of collagen molecules, which are in vivo hydrolysed by collagenase and matrix metalloproteinase enzymes. This process may be regulated by cross-linking and structural modification of the final ADMs. Products of degradation of ADMs are collagen type I and III, which induce chemotactic infiltration by fibroblasts $(57,58)$. Therefore, a controlled rate of biodegradation, non-toxic metabolites, low or absent antigenicity, inflammatory or foreign body reactions are mandatory (54).

Resistance to shear forces

ADMs should be strong enough to be held in a place of difficult anatomic areas and should resist shear forces (53). ADMs use is an appropriate way to minimize scar contracture and to improve the quality of the grafted area in strained regions requiring elasticity, pliability, and stability.

\section{Summary of the desired properties of ADMs}

The ideal biologic dermal substitute should have the following characteristics, enumerated for the most part by Pruitt and Levine more than twenty years ago (30), and added to by others should include (31):

- absence of antigenicity,

- tissue compatibility,

- absence of local or systemic toxicity,

- impermeability to exogenous microorganisms,

- water vapor transmission similar to normal skin,

- rapid and sustained adherence to wound surface,

- conformity to surface irregularities,

- elasticity to permit motion of underlying tissue,

- resistance to linear and shear stresses,

- tensile strength to resist fragmentation,

- inhibition of wound surface flora and bacteria,

- biodegradability,

- reducing time to heal,

- not increasing the rate of infection,

- minimizing patient discomfort,

- minimizing nursing care of a wound,

- patient acceptance,

- low cost,

- long shelf life, minimal storage requirements (3).

\section{Conclusions}

Although ADMs do not fulfil all of the requirements for an ideal dermal substitute, they play an important role in cases of severe skin losses or large and deep wounds, and in other situations in which healing by primary intention or autografting are not possible anymore. With further research, their applications have been advanced for diverse indications in soft tissue reconstructions. Early revascularization of allografts reduces bacterial contamination. It was proven histologically, that matrices with a preserved native ultrastructure of ECM showed a higher rate of cell infiltration, fibroblasts deposition and revascularization. Research and clinical development of new generation of acellular matrices with incorporated cultured cells will likely yield new products and give new hope for continued improvements in functional and cosmetic outcomes.

\section{References}

1. Shahriar S, Arno A, Jeschke MG. The Use of Dermal Substitutes in Burn Surgery: Acute Phase. Wound Repair Regen 2014; 22 (1). doi: 10.1111/wrr.12119.

2. Sanon S, Hart DA, Tredget EE. Molecular and Cellular Biology of Wound Healing and Skin Regeneration. In: Albanna MZ, Holmes IV JH. Skin Tissue Engineering and Regenerative Medicine, Academic Print in an inprint of Elsevier, 2016, s. 19-38.

3. Ehrenreich M, Ruszczak Z. Update on Dermal Substitutes. 2006. Acta Dermatovenerol Croat 2006; 14 (3): 172-187.

4. Lee KH. Tissue-engineered human living skin substitutes: Development and clinical application. Yonsei Med J 2000; 41 (6): 774-779.

5. Pham C, Greenwood J, Cleland H, Woodruff P, Maddern G. Bioengineered skin substitutes for the management of burns: A systematic review. Burns 2007; 33: 946-957.

6. Zuijlen van PP, Angeles AP, Kreis RW, Bos KE, Middelkoop E. Scar assessment tools: implications for current research. Plast Reconstr Surg 2002; 109 (3): 1108-1122.

7. Van Der Veen VC, van der Wal MBA, van Leuwen MCE, Ulrich MMW, Middelkoop E. Biological background of dermal substitutes. Burns. 2010; 36: 305-21.

8. Hodgkinson T, Bayat A. Dermal substitute-assisted healing: enhancing stem cell therapy with novel biomaterial design. Arch Dermatol Res 2011; 303: 301-315.

9. Koller J. Popáleniny (Burns). Bratislava: Univerzita Komenského, 2010, s. 76

10. Bush K, Gertzman AA. Process Development ad Manufacturing of Human and Animal Acellular Dermal Matrices. In: Albanna MZ, Holmes IV JH. Skin Tissue Engineering and Regenerative Medicine, Academic Print in an inprint of Elsevier, 2016.

11. Bush K, Gertzman AA. Process Development and Manufacturing of Human and Animal Acellular Dermal Matrices. In: Albanna MZ, Holmes IV JH. Skin Tissue Engineering and Regenerative Medicine, Academic Print in an inprint of Elsevier, 2016, s. 83-103.

12. Walter R, Matsuda T, Reyes H, Walter J, Hanumadass M. Characterization of acellular dermal matrices (ADMs) prepaared by two different methods. Burns 1998; 24 (2): 104-113. 
13. Truong AN, Kowal-Vern A, Latenser BA, Wiley DE,Walter RJ. Comparision of dermal substitutes in wound healing utilizing a nude mouse model. J Burns Wounds 2005; 4: 72-82.

14. Gilberrt TW. Strategies for tissue and organ decelullarization. J Cell Biochem March 2012; 113 (7): 2217-2222.

15. Hrebikova H, Diaz D, Mokry J. Chemical decellularitaion: a promisisng approach foe preparation of extrcellular matrix. Biomed Pap med Fac Univ Palacky Olomouc Czech Repub 2013; 157: 1-6.

16. Reing J, Brown B, Daly K, Freund J, Gilbert T, Hsiong $S$ et al. The effects of processing methods upon mechanical and biologic properties of porcine dermal extracellular matrix scaffolds. Biomaterials 2011; 31 (33): 8626-8633.

17. Crapo PM, Gilbert TW, Badylak SF. An overvieew of tissue and whole organ decellularization processes. Biomaterials 2011; 32 (12): 3233-3243.

18. Dragúňová J, Kabát P, Babál P et al. Development of a new method for the preparation of an acellular allodermis, quality control and cytotoxicity testing. Cell Tissue Bank (2017) 18: 153. https://doi.org/10.1007/ s10561-017-9625-6.

19. Vitteková M, Dragúňová J, Kabát P, Božíková M, Bakoš D, Koller J. Cytotoxicity testing of scaffolds potentially suitable for the preparation of three-dimensional skin substitutes. Cell Tissue Bank 2014; 15 (3): 345-355. doi:10.1007/s10561-013-9390-0.

20. Faulk DM, Badylak SF. Natural Biomaterials for Regenerative Medicine Applications. In Guiseppe Orlando, Regenerative Medicine Applications in Organ Transplantation. Academic Print in an inprint of Elsevier, 2013: 1050 (101-112).

21. Han JG et al. Histologic Analysis of Acellular Dermal Matrix in the Treatment of Anal Fistula in an Animal Model. J Amer Coll Surg 2009; 208 (6): 1099-1106. https://doi.org/10.1016/j.jamcollsurg.2009.02.052.

22. Wainwright DJ. Use of an acellular allograft dermal matrix (AlloDerm) in the management of full-thickness burns. Burns 1995; 21 : 243-248.

23. Yang Z, Wu X, Chen C, Huang Y, Fang L, Li X, Zhang Y, Jia M. Comparison of type I tympanoplasty with acellular dermal allograft and cartilage perichondrium. Acta Oto-Laryngol 139: 10: 833-836. doi: 10.1080/00016489.2019.1637541.

24. Shi LJ, Wang Y, Yang C, Jiang WW. Application of Acellular Dermal Matrix in Reconstruction of Oral Mucosal Defects in 36 Cases. J Oral Maxillofac Surg 2012; 70 (11): e586-e591. https://doi.org/10.1016/j. joms.2012.07.024.

25. Yde SS, Brunbjerg ME, Gudmundsdottir G, Bazys M, Heje M, Damsgaard TE. Dural repair using porcine ADM: two cases and a literature review. Case Rep Plast Surg Hand Surg 2017; 4 (1): 5-8. doi: 10. 1080/23320885.2016.1278169.

26. Kridel RWH, Foda H, Lunde KC. Septal Perforation Repair With Acellular Human Dermal Allograft. Arch Otolaryngol Head Neck Surg 1998; 124 (1): 73-78. doi: https://doi.org/10.1001/archotol.124.1.73.

27. Wood B, Mantilla-Rivas E, Goldrich A, Boyajian MK, Oh A, Rogers GF, Boyajian MJ. Correction of Microstomia Reconstruction with the Use of Acellular Dermal Matrix for Buccal Reconstruction. J Craniofac Surg 2019; 30 (3): 736-738. doi: 10.1097/SCS.0000000000005182.

28. Butler CE, Langstein HN, Kronowitz SJ. Pelvic, abdominal, and chest wall reconstruction with AlloDerm in patients at increased risk for mesh-related complications. Plast Reconstr Surg 2005; 116: 1263-1277.
29. Ceresoli M, Coccolini F, Ansaloni L, Sartelli M, Campanelli G, Catena F. Biological Prosthesis for Abdominal Wall Reconstruction. In: Coccolini F, Ivatury R, Sugrue M, Ansaloni L (Eds). Open Abdomen. Hot Topics in Acute Care Surgery and Trauma. Springer, Cham, 2018. https:// doi.org/10.1007/978-3-319-48072-5_20.

30. Hoang D, Steven P, Chen VW, Stasiak A, Cohen M, Kulber DA. Use of Acellular Dermal Matrix Following Fasciectomy for the Treatment of Dupuytren's Disease (Plast Reconstr Surg Glob Open 2019; 7: e2263. doi: 10.1097/ GOX.0000000000002263.

31. Winters CL, Brigido SA, Liden BA et al. A multicenter study involving the use of a human acellular dermal regenerative tissue matrix for the treatment of diabetic lower extremity wounds. Adv Skin Wound Care 2008; 21: 375e381.

32. Cuomo R, Grimaldi L, Brandi C, Sisti A, Nisi G. Vaginoplasty: What's New From 1946 to Date Commentary on: Vaginoplasty with Acellular Dermal Matrix after Radical Resection for Carcinoma of the Uterine Cervix. J Invest Surg 2019; 32: 2: 186-188. https://doi.org/10.1080/089 41939.2017.1411544.

33. D'Angelo W, Dziki J, Badylak S. The challenge of stress incontinence and pelvic organ prolapse revisiting biologic mesh materials. Curr Opinion Urol 2019; 29 (4): 437-442. doi: 10.1097/MOU.0000000000000645.

34. Ayshford CA, Shykhon M, Uppal HS, Wake M. Endoscopic repair of nasal septal perforation with acellular human dermal allograft and an inferior turbinate flap. Clin Otolaryngol 2003; 28: 29-33.

35. Maas CS, Papel ID, Greene D, Stoker DA. Complications of injectable synthetic polymers in facial augmentation. Dermatol Surg 1997; 23: 871-877.

36. Mendenhall SD, Schmucker RW, Daugherty THF, Kottwitz KM, Reichensperger JD, Koirala J, Cederna PS, Neumeister MW. A Microbiological and Ultrastructural Comparison of Aseptic versus Sterile Acellular Dermal Matrix as a Reconstructive Material and a Scaffold for Stem Cell Plast Reconstr Surg 2017; 140: 97. doi: 10.1097/PRS.0000000000003448).

37. Lindberg K, Badylak SF. Porcine small intestinal submucosa (SIS): a bioscaffold supporting in vitro primary human epidermal cell differentiation and synthesis of basement membrane proteins. Burns 2001; 27: $254 \mathrm{e} 266$.

38. Hu Z, Zhu J, Cao X, Chen C, Li S, Guo D, Zhang J, Liu P, Shi F, Tang B. Composite Skin Grafting with Human Acellular Dermal Matrix Scaffold for Treatment of Diabetic Foot Ulcers: A Randomized Controlled Trial. 2016. J Am Coll Surg 2016; 222: 1171e1179.

39. Balasubramani M et al. Skin substitutes: a review. Burns 2001; 27 : 534-544.

40. Carlsson AH, Gronet EM, Rose LF, Chan R. Clinical Applications of Acellular Dermal Matrices. In Reconstructive Surgery. In: Albanna MZ, Holmes IV JH. Skin Tissue Engineering and Regenerative Medicine, Academic Print in an inprint of Elsevier, 2016.

41. Malcarney HL, Bonar F, Murrell GA. Early inflammatory reaction after rotator cuff repair with a porcine small intestine submucosal implant: a report of 4 cases. Am J Sports Med 2005; 33: 907e911.

42. Zheng MH, Chen J, Kirilak $Y$ et al. Porcine small intestine submucosa (SIS) is not an acellular collagenous matrix and contains porcine DNA: possible implications in human implantation. J Biomed Mater Res B Appl Biomater 2005; 73: 61e67.

43. Schultz GS, Sibbald RG, Falanga $V$ et al. Wound bed preparation: a systematic approach to wound management. Wound Repair Regen 2003; 11 (Suppl 1): S1eS28. 


\section{6-394}

44. Jeon H, Kim J, Yeo $\mathbf{H}$ et al. Treatment of diabetic foot ulcer using matriderm in comparison with a skin graft. Arch Plast Surg 2013; 40: $403 \mathrm{e} 408$.

45. Iorio ML, Goldstein J, Adams M et al. Functional limb salvage in the diabetic patient: the use of a collagen bilayer matrix and risk factors for amputation. Plast Reconstr Surg 2011; 127: 260e267.

46. Stacey DH. Use of an Acellular Regenerative Tissue Matrix Over Chronic Wounds. Eplasty 2013; 13 (e61): 514-521.

47. Suh H, Hong PJ. One stage allogenic acellular dermal matrices $(\mathrm{ADM})$ and split-thickness skin graft with negative pressure wound therapy. In Maduri Gore, Skin grafts (2013). InTech http://dx.doi. org/10.5772/5330.

48. Chun Y, Verma K, Rosen H, Lipsitz S, Morris D, Kenney P, Eriksson E. Implant-Based Breast Reconstruction Using Acellular Dermal Matrix and the Risk of Postoperative Complications. Plast Reconstr Surg 2010; 125 (2): 429-436. doi:0.1097/PRS.0b013e3181c82d90.

49. Piccoli M, Agresta F, Attina GM, Amabile D, Marchi D. "Complex abdominal wall" management: evidence-based guidelines of the Italian Consensus Conference. In: Updates in Surgery 2018. https://doi. org/10.1007/s13304-018-0577-6.

50. FitzGerald JF, Kumar AS. Biologic versus synthetic mesh reinforcement: what are the pros and cons? Clin Colon Rectal Surg 2014; 27: 140 148. doi: 10.1055/s-0034-1394155.

51. Dasgupta A, Orgill D, Galiano RD et al. A Novel Reticular Dermal Graft Leverages Architectural and Biological Properties to Support Wound Repair. Plast Reconstr Surg Global Open 2016; 4 (10): e1065. doi:10.1097/ GOX.0000000000001065.
52. Klein GM, Singh G, Marquez J, Gebre M, Barry R, Huston TL, Ganz JC, Khan SU, Dagum AB, Bui DT. Acellular Dermal Matrix Sterility: Does It Affect Microbial and Clinical Outcomes Following Implantation? Plast Reconstr Surg Glob Open 2019; 7: e2355. doi: 10.1097/ GOX.0000000000002355.

53. Leventhal D, Furr M, Reiter D. Treatment of keloids and hypertrophic scars. Arch Facial Plast Surg 2006; 8: 362-368.

54. Alisky JM. Xenografts are an achievable breakthrough. Med Hypotheses 2004; 63: 92-97.

55. Burke JF, Yannas IV, Quinby WC Jr, Bondoc CC, Jung WK. Successful use of a physiologically acceptable artificial skin in the treatment of extensive burn injury. Ann Surg 1981; 194: 413-428.

56. Richters CD, Pirayesh A, Hoeksema H, Kamperdijk WA, Kries RW, Dutrieux RP, Monstrey S, Hoekstra MJ. Development of a dermal matrix from glycerol preserved allogeneic skin. Cell Tissue Banking 2008; 9: 309-315.

57. Parenteau-Bareil R, Gauvin R, Berthod F. Collagen-Based Biomaterials for Tissue Engineering Applications. Materials 2010; 3 (3): 1863-1887. doi: 10.3390/ma3031863.

58. Hu Y et al. Development of collagen/polydopamine complex matrix as mechanically enhanced and highly biocompatibile semi-natural tissue engineering scaffold, Acta Biomaterialia 2017; 47: 135. doi: 10.1016/j. actbio.2016.10.017.

59. Pruitt BA, Levine NS. Characteristics and uses of biologic dressings and skin substitutes. Arch Surg 1984; 119: 312-322.

60. Smith DJ Jr. Use of Biobrane in wound management. J Burn Care Rehabil 1995; 16: 317-320. 